\title{
Effects of Water Hardness on Textile Detergency Performance in Aqueous Cleaning Systems
}

\author{
Keiko Gotoh ${ }^{1 *}$, Kaori Horibe ${ }^{1}$, Yang Mei ${ }^{1}$ and Toshiyuki Tsujisaka ${ }^{2}$ \\ ${ }^{1}$ Faculty of Human Life and Environment, Nara Women's University (Kita-uoya-nishi-machi, Nara 630-8506, JAPAN) \\ ${ }^{2}$ Nara Prefecture Institute of Industrial Development (129-1 Kashiwagi-cho, Nara 630-8031, JAPAN)
}

\begin{abstract}
The effects of water hardness on textile detergency in aqueous solutions were systematically investigated using four surfactants: sodium oleate (OLNa), linear dodecylbenzene sulfonate (LAS), sodium dodecyl sulfate (AS), and polyoxyethylene (10) dodecyl ether (AE). Water hardness was adjusted according to the standard procedure described in IEC 60734:2012. As expected, by adding hardness salts the surface tension of the OLNa solution increased. Surprisingly, the addition of hardness salts lowers the surface tension for the LAS and AS solutions. In the case of the AE solution, hardness salt did not affect the surface tension. A decrease in transmittance and foamability after adding hardness salts was observed for every anionic surfactant solution, indicating that anionic surfactants can combine with divalent ions to form insoluble precipitates. Detergency experiments were performed using cotton plain-woven and towel fabrics soiled with a carbon black and oleic acid mixture. One piece each of untreated and soiled fabric were stacked and placed horizontally in detergent solution with or without hardness salts. As a mechanical action of soil removal, the shaking of $190 \mathrm{spm}$ was applied. Soil removal and redeposition due to washing were evaluated from changes in values of the Kubelka-Munk function for both fabrics. With increasing water hardness, soil removal decreased and redeposition increased. In order of decreasing detergency, the surfactants were as follows: LAS > OLNa $\approx \mathrm{AS}>\mathrm{AE}$. The results indicate that precipitates, formed by reaction of LAS or AS with hardness salts, are strongly adsorbed on the water surface because of their hydrophobicity, but they have no detergency power. The field emission scanning electron microscopic observation and $\mathrm{X}$-ray photoelectron spectroscopic analysis showed that $\mathrm{Ca}(\mathrm{LAS})_{2}$ precipitation clung to fiber surfaces, and remained on the surfaces after washing. Significant changes in the cotton fabric due to washing were observed in mechanical properties and water absorbency.
\end{abstract}

Key words: water hardness, surfactant, cotton, detergency, fabric damage

\section{INTRODUCTION}

It is well known that hardness of laundry water affects the detergency performance of surfactants. Soaps and detergents react with calcium and magnesium in hard water to form insoluble precipitates, and hence larger amounts of soap and detergents are needed for cleaning in hard water than in soft water ${ }^{1)}$. In addition, the insoluble precipitates formed cling to fibers, and remain in the fabric after laundering $^{2}$. This makes the fabric stiff and rough, and causes colors to fade ${ }^{3,4)}$.

Many researchers have reported the effect of water hardness on the detergency power of surfactants. Matheson and co-workers have investigated the interaction between linear alkylbenzene sulfonate (LAS) and calcium ions $^{5-7)}$. They found that the addition of micelle promoting agents improved the detergency performance of LAS in hard water by reducing sensitivity to water hardness. Foam formation and the detergency performance of LAS solutions in the presence of calcium have been studied by Cohen et $a l .{ }^{8,9)}$. They also reported that detergency performance in the presence of magnesium is significantly higher than with calcium ${ }^{10)}$. Goto-Doshida et al. studied the relation between detergency and calcium on the cotton fabric, or in the wash bath, in the presence of anionic surfactants, nonionic surfactants, and builders ${ }^{11,12)}$. They found that the calcium content in cotton fabrics had no influence on detergency at high water hardness, and a negative influence at lower water hardness. With respect to the commercial laundry detergent, Brown et al. investigated the effect of water hardness on washing quality ${ }^{13)}$. They found that de-

\footnotetext{
*Correspondence to: Keiko Gotoh, Faculty of Human Life and Environment, Nara Women's University, Kita-uoya-nishi-machi, Nara 630-8506, JAPAN

E-mail: k-gotoh@cc.nara-wu.ac.jp

Accepted November 18, 2015 (received for review July 14, 2015)

Journal of Oleo Science ISSN 1345-8957 print / ISSN 1347-3352 online

http://www.jstage.jst.go.jp/browse/jos/ http://mc.manusriptcentral.com/jjocs
} 
tergent containing a non-ionic surfactant with a phosphate builder gave the best whiteness results, regardless of water hardness. It is commonly said that liquid detergents wash equally well in both soft and hard water, and powdered detergents wash better than liquids in soft water ${ }^{1)}$.

Water hardness is a key factor in the success of the washing process in areas of the world where hard water is present $^{14)}$. However, the effects of water hardness on precipitate formation, foamability, surface tension, detergency, and properties of the washed fabric using some common surfactant species have not been systematically examined in the same experimental system. In the present study, detergency and soil redepostion at different levels of water hardness, using solutions of sodium oleate (OLNa), linear dodecylbenzene sulfonate(LAS), sodium dodecyl sulfate (AS), or polyoxyethylene (10) dodecyl ether (AE) as surfactants were evaluated using artificially soiled cotton fabrics. The results are discussed in terms of solution properties of the surfactants. Additionally, the cotton fiber surface after washing was examined by scanning electron microscopy (SEM) and X-ray photoelectron spectroscopy (XPS). Water transfer and mechanical properties of the cotton fabrics after washing were also investigated, in the presence or absence of hardness salts, from the viewpoint of the sensorial comfort of textiles.

\section{EXPERIMENTAL PROCEDURES}

\subsection{Materials}

The cotton fabrics used were plain-woven (10A, wfk Testgewebe GmbH, Germany) or towel (220 monme, Toshin). Both fabrics were purified twice in boiling water, and then cut into swatches $50 \times 50 \mathrm{~mm}^{2}$ after drying in air. For contact angle measurement, a cellulose film $20 \mu \mathrm{m}$ in thickness was used, and was provided by Rengo Co., Ltd., Japan. We chose oleic acid (Wako Pure Chemical Industries, Ltd., Japan) and carbon black (Sentakukagaku-kyokai, Japan) as model soils.

Sodium oleate, linear dodecylbenzene sulfonate, sodium dodecyl sulfate, and polyoxyethylene (10) dodecyl ether were used as surfactants. OLNa, LAS and AS were commercially available from Wako Pure Chemical Industries, Ltd., Japan, and AE was provided by Lion Corporation, Japan. Calcium carbonate, magnesium sulfate and sodium hydrogen carbonate of extra-pure grade were added to the detergent solution to control water hardness according to IEC 60734:2012.

\subsection{Measurements of surfactant solution properties}

Transmittance of the detergent solution was measured in a rectangular optical borosilicate glass cell $(10 \times 10 \times 43$ $\mathrm{mm}^{3}$ ) using a spectrophotometer (U-1100, Hitachi Science Systems, Ltd.) at a wavelength of $550 \mathrm{~nm}$.
The foaming power of the detergent solution was determined according to the Ross-Miles method, in which foam is made by letting a standard volume of liquid fall on top of another volume of liquid(ASTM: D1173-53:2001) ${ }^{15)}$. After falling $0.02 \mathrm{dm}^{3}$ of the detergent solution from the head of volumetric pipette into a graduated cylinder containing $0.01 \mathrm{dm}^{3}$ of the same detergent solution, the foam height was immediately measured. The transmittance and foaming power measurements were carried out in a room maintained at $25 \pm 1^{\circ} \mathrm{C}$.

Surface tension measurement of the detergent solution was performed by the pendant drop method, using a video contact angle system equipped with a CCD camera(VCA 2500, AST Products Inc., USA). The detergent solution was dispensed from a microsyringe to form a pendant drop, which was made as large as possible without letting it fall from the tip of the needle. Surface tension was determined by image analysis through video-image digitization and numerical curve fitting using the Laplace equation of capillarity $^{16)}$.

The contact angle of the detergent solutions on cellulose film, as a model cotton substrate, was measured by the sessile drop technique using the same video contact angle system. The contact angle immediately after placing a $2-3-\mu \mathrm{L}$ drop on the film was determined as the approximate value of the advancing angle ${ }^{17)}$. The surface tension and the contact angle measurements were repeated 5 to 10 times for the same detergent solution in a room maintained at $20^{\circ} \mathrm{C}$ and $65 \% \mathrm{RH}$.

\subsection{Preparation of artificially soiled fabrics}

As a soil bath, a solution containing $0.1 \mathrm{dm}^{3}$ ethanol, $5 \mathrm{~g}$ oleic acid, and $0.03 \mathrm{~g}$ carbon black was prepared by ultrasonication. A piece of cotton fabric was then immersed in the soil bath, while sonicating to allow the soil to deposit onto the fabric uniformly. After soiling for $30 \mathrm{~s}$, the fabric was dried and stored in a refrigerator for $2 \mathrm{~d}$ prior to washing.

Immediately prior to washing, the Kubelka-Munk function values $(\mathrm{K} / \mathrm{S})$ of the original and soiled fabrics were measured using a high-precision spectrophotometer (SE 6000, Nippon Denshoku, Japan) by stacking four layers of the same type of fabric. To measure reflectance, the halogen illumination/light receiving conditions were a $0^{\circ}$ illumination and $45^{\circ}$ circumference light receiving system, which was adopted for the spectrophotometer. Reflectance of each side of the fabric was read at the wavelength of 460 $\mathrm{nm}$ using a $30 \mathrm{~mm} \Phi$ of measuring aperture, and the final value was obtained as the average.

\subsection{Washing test and detergency evaluation}

A piece each of soiled and untreated fabric were stacked with the soiled fabric on top, and placed horizontally in a $0.2 \mathrm{dm}^{3}$ glass vessel containing the aqueous detergent solu- 
tion in a bath ratio of 1:50. The surfactant concentration of the detergent solution was adjusted to critical micelle concentration (cmc), obtained in the absence of hardness salt, as described in Section 3.1. To apply mechanical action for soil removal, a shaking action of 40-mm-stroke at $190 \mathrm{spm}$ was applied using a vibrating shaker(BW201, Yamato Science Co., Ltd, Japan). The wash temperature was controlled at $25 \pm 1^{\circ} \mathrm{C}$ in a water bath using a throw-in type low heating water circulator (BF400, Yamato Science Co., Ltd, Japan). After washing for $10 \mathrm{~min}$, the fabric was rinsed for $60 \mathrm{~s}$ in $0.1 \mathrm{dm}^{3}$ of water with the same hardness as the detergent solution.

The K/S value of the washed fabric was measured again as described in Section 2.3. Detergency and soil redeposition were calculated by the following equations ${ }^{18)}$ :

$$
\begin{aligned}
& \text { Soil removal }(\%)=\frac{(\mathrm{K} / \mathrm{S})_{\mathrm{s}}-(\mathrm{K} / \mathrm{S})_{\mathrm{w}}}{(\mathrm{K} / \mathrm{S})_{\mathrm{s}}-(\mathrm{K} / \mathrm{S})_{\mathrm{o}}} \times 100 \\
& \text { Soil redeposition }=\frac{(\mathrm{K} / \mathrm{S})_{\mathrm{ow}}-(\mathrm{K} / \mathrm{S})_{\mathrm{o}}}{(\mathrm{K} / \mathrm{S})_{\mathrm{s}}-(\mathrm{K} / \mathrm{S})_{\mathrm{w}}}
\end{aligned}
$$

where the subscripts $\mathrm{s}$ and $\mathrm{w}$ refer to the soiled fabrics before and after the washing, respectively, and the subscripts ow and o refer to the original untreated fabrics before and after washing, respectively.

The washing tests were repeated 2 to 6 times under the same experimental conditions, and standard deviation was within $\pm 10 \%$.

\subsection{Surface characterization of cotton fabric}

Field emission scanning electron microscopic (FE-SEM) observation of the original fabrics before and after washing was performed using a scanning electron microscope (Hitachi High-Technologies Corporation SU8020) with 0.7 or $1.0 \mathrm{kV}$ accelerating voltage and $20 \mathrm{~s}$ scan speed, at a magnification power of 1000. Prior to observation, the specimens $\left(5 \times 5 \mathrm{~mm}^{2}\right)$ were mounted with carbon tape to prevent the accumulation of electrostatic surface charge. Topographical information and compositional contrast were obtained by detecting selective low angle backscattered electrons.

XPS was performed using an Axis-Ultra DLD spectrometer (Kratos, UK) fitted with a monochromatic $\mathrm{Al} \mathrm{K}_{\alpha}$ radiation at $1486.7 \mathrm{eV}(75 \mathrm{~W})$ with charge neutralization. Highresolution spectra of the core levels of $\mathrm{Mg} 1 \mathrm{~s}, \mathrm{Na} 1 \mathrm{~s}, \mathrm{Ca} 2 \mathrm{p}$ and S2p were acquired with pass energy of $20 \mathrm{eV}$ and a slot aperture of $0.3 \times 0.7 \mathrm{~mm}^{2}$. Spectra were collected at a photoelectron take off angle of 90 degrees. Pressure in the analytical chamber was maintained near $10^{-8} \mathrm{~Pa}$. All XPS binding energies were referenced to the C1s peak of adventitious carbon at a binding energy of $284.8 \mathrm{eV}$. The collected spectra were deconvoluted with a Gaussian-Lorentzian approximation, after Shirley background subtraction, using Vision 2 software.

\subsection{Evaluation of cotton towel properties}

Wicking of water in the capillaries of the cotton towel was evaluated by the Byreck Method (JIS L1907:2004). A cotton towel strip $25 \mathrm{~mm}$ in width and $200 \mathrm{~mm}$ in length was hung vertically, and the lower $10 \mathrm{~mm}$ was dipped into water containing Sirius Red $4 \mathrm{~B}$ at a concentration of 0.02 $\mathrm{g} / \mathrm{dm}^{3}$. The height of water due to capillary action was measured after 10 min above water level at the center of the strip.

Cantilever bending resistance was evaluated according to JIS L1096:2007. In the test, a horizontal strip of cotton towel $20 \mathrm{~mm}$ in width and $150 \mathrm{~mm}$ in length slid in a direction parallel to its long dimension at a specified rate until the leading edge projected from the edge of a horizontal surface. The length of overhang was measured when the tip of the specimen was depressed under its own weight to the point where a line joining the tip to the edge of the platform made a $45^{\circ}$ angle with the horizontal.

Kawabata Fabric Evaluation System (KES-FB, Kato Tech Co. Ltd.) was used for evaluating fabric mechanical properties. Linearity of the load-extension curve(LT), bending rigidity (B), shear stiffness $(G)$, linearity of compressionthickness curve (LC), and coefficient of friction (MIU) were evaluated. For evaluation of surface friction, a $10 \times 10 \mathrm{~mm}^{2}$ piano wire sensor was used as a contact friction sensor. Evaluation of cotton towel properties was carried out in a room maintained at $20^{\circ} \mathrm{C}$ and $65 \% \mathrm{RH}$.

\section{RESULTS AND DISCUSSION}

\subsection{Effect of water hardness on surface tension}

Surface tension vs the logarithm of surfactant concentration curves, in the presence or absence of hardness salts, are shown in Fig. 1. In all cases, the surface tension decreased linearly with increasing concentration and retained an almost constant value above the cmc.

For OLNa, the surface tension below the cmc increased by the addition of hardness salts, indicating a decrease in bulk OLNa concentration due to formation of insoluble precipitates. As a result, the cmc increased with increasing water hardness. On the other hand, the surface tension decreased by the addition of hardness salts in the cases of LAS and AS, suggesting the adsorption of insoluble precipitates onto the water surface. Especially for LAS, both cmc and the surface tension above the cmc decreased with increasing water hardness. This considerable decrease in the cmc of LAS by adding $\mathrm{Ca}^{2+}$ and $\mathrm{Mg}^{2+}$ has previously been reported by other investigators ${ }^{19,20)}$. The decrease in the surface tension above the cmc suggests a reduction of the occupied water surface area per molecule. This may be caused by a significant restriction in the packing of LAS molecules in the presence of $\mathrm{Ca}^{2+}$ and $\mathrm{Mg}^{2+}$ ions ${ }^{20)}$. It has also been reported that the presence of hardness salts 

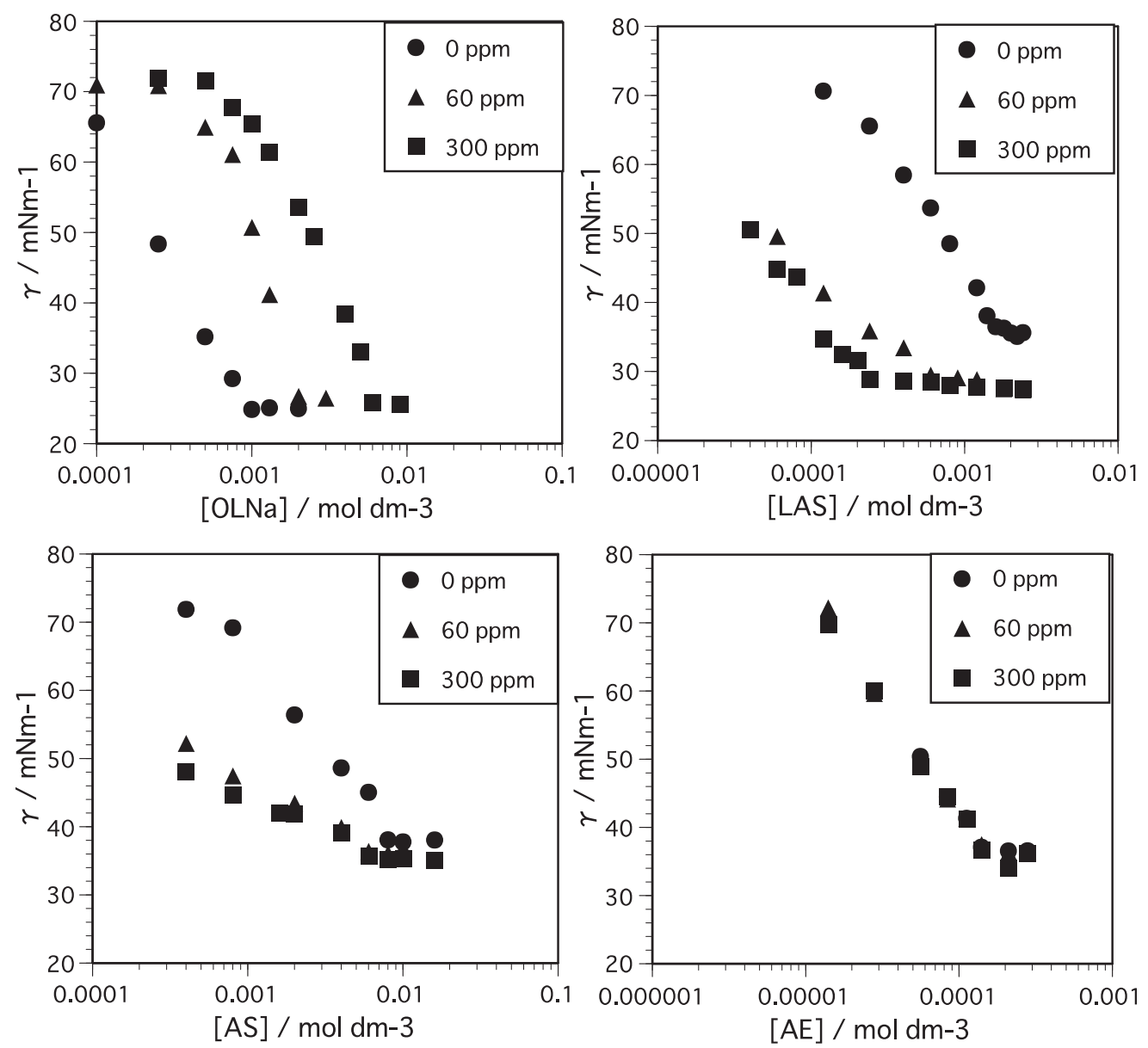

Fig. 1 Surface tension, $\gamma$, vs. logarithm concentration curves for four surfactant solutions with and without hardness salts.

results in an effective increase of the SDS adsorption activ$i^{21)}$. In contrast to the anionic surfactants, the water hardness had little effect on the surface tension of nonionic AE solutions.

The effects of water hardness on the transmittance and foamability of the surfactant solution were determined, at the cmc obtained in the absence of hardness salt, as shown in Fig. 2. Little effect was observed for nonionic AE. Transmittance of the anionic surfactant solutions decreased with increasing water hardness as a result of the formation of insoluble precipitates, and this was especially evident for LAS. This indicates that LAS combines with divalent ions due to specific interactions, as well as electrostatic binding $^{20)}$.

The foaming power of the anionic surfactant solutions significantly decreased in the presence of hardness salts. This was especially evident for OLNa, where no foam was observed at 300 ppm hardness. For the LAS and AS solutions, the reduction of foaming power by adding hardness salts was not as evident. Combined with reduction in surface tension shown in Fig. 1, we believe that the insoluble precipitates formed by the reaction of LAS or AS with hardness salts are strongly adsorbed at the water surface.

\subsection{Effect of hardness salts on soil removal and redeposi- tion}

The results of the washing tests as a function of water hardness are presented in Figs. 3 and 4. In the case of the cotton woven fabric in the anionic surfactant solutions (Fig. 3), soil removal from the soiled fabric decreased and redeposition increased onto the original untreated fabric at increasing water hardness. For LAS, the detergency power was remarkably weakened by the addition of hardness salts. On the other hand, little influence of hardness on the detergency power was observed in the case of the nonionic surfactant, AE.

The detergency power with the cotton towel fabric (Fig. 4) showed a similar dependence with respect to water hardness, although soil removal and redeposition were greater when compared to the cotton woven fabric. Images of the cotton towel fabrics before and after washing in the LAS solutions at water hardness of 0, 60, and $300 \mathrm{ppm}$ are presented in Fig. 5. After washing in the LAS solution at 300 ppm, we observed that much soil remained or was re- 

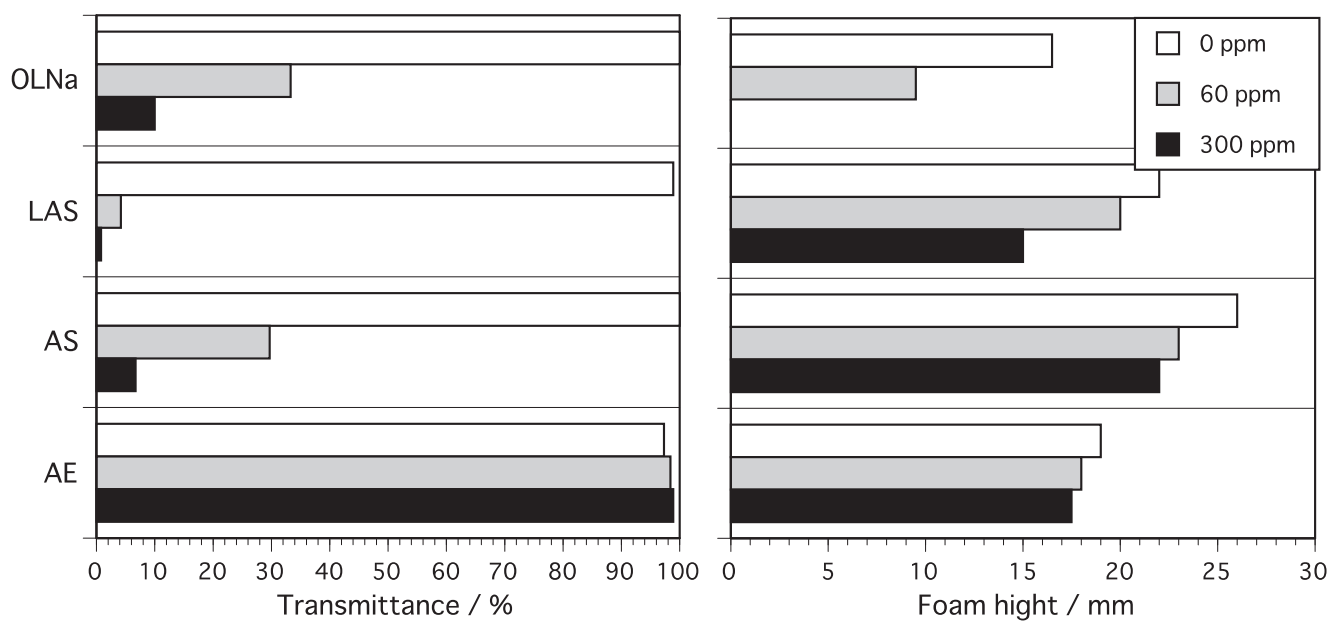

Fig. 2 Transmittance and foamability of the surfactant solutions with and without hardness salts used for the washing test.
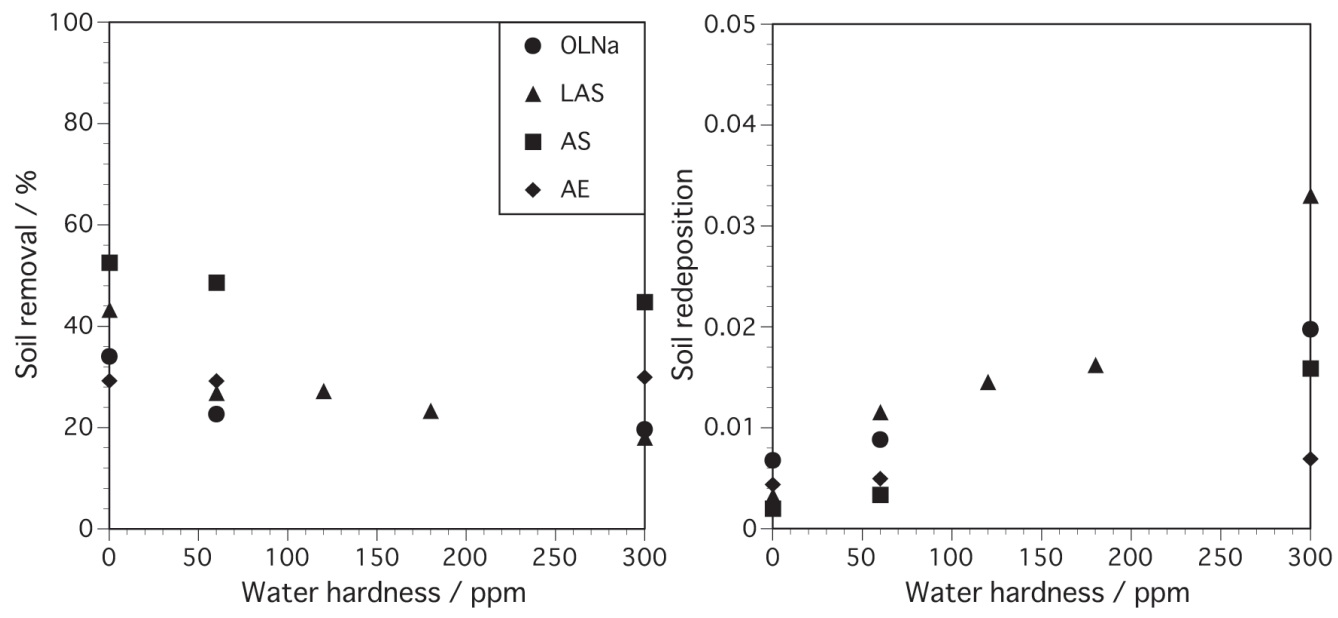

Fig. 3 Effects of water hardness on soil removal and redeposition in four surfactant solutions for cotton woven fabrics.
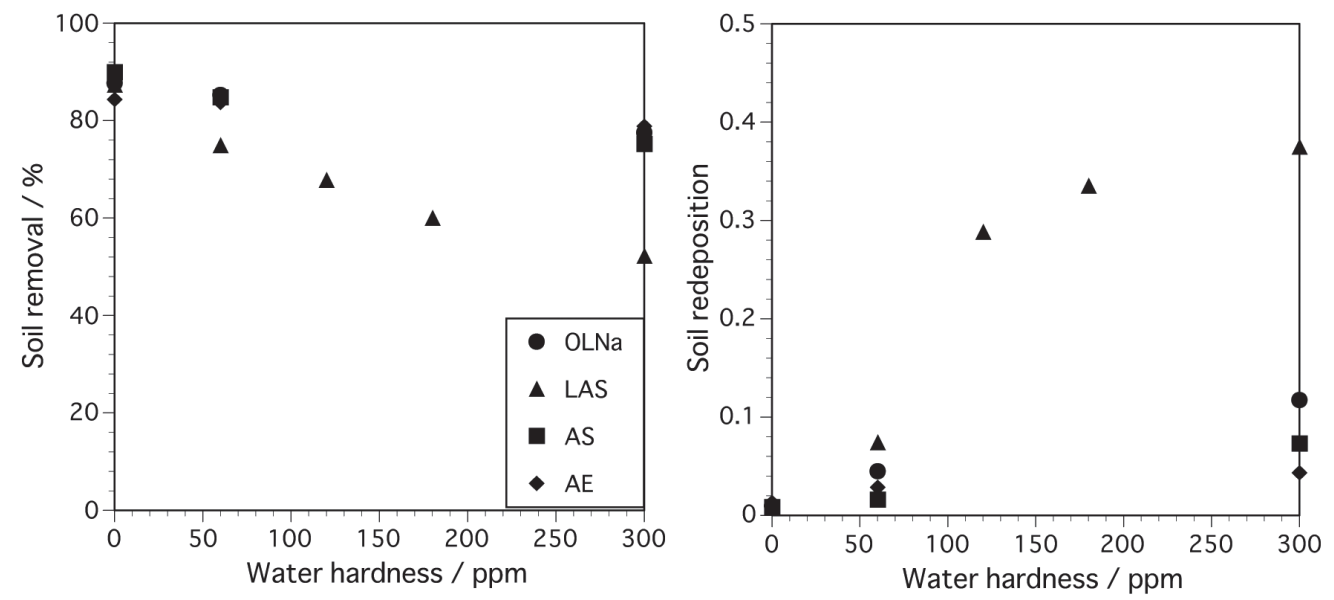

Fig. 4 Effects of water hardness on soil removal and redeposition in four surfactant solutions for cotton towel. 
Soiled fabric
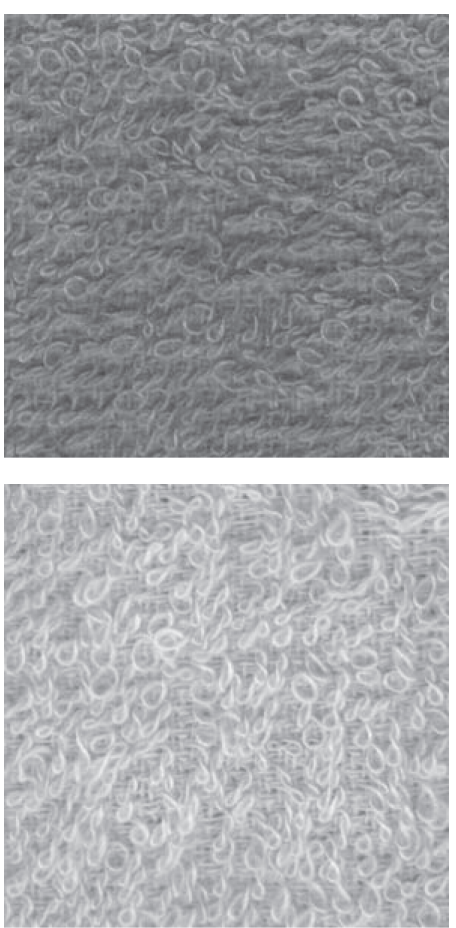

\section{After washing} at $0 \mathrm{ppm}$

\section{After washing at $60 \mathrm{ppm}$}

\section{After washing} at $300 \mathrm{ppm}$
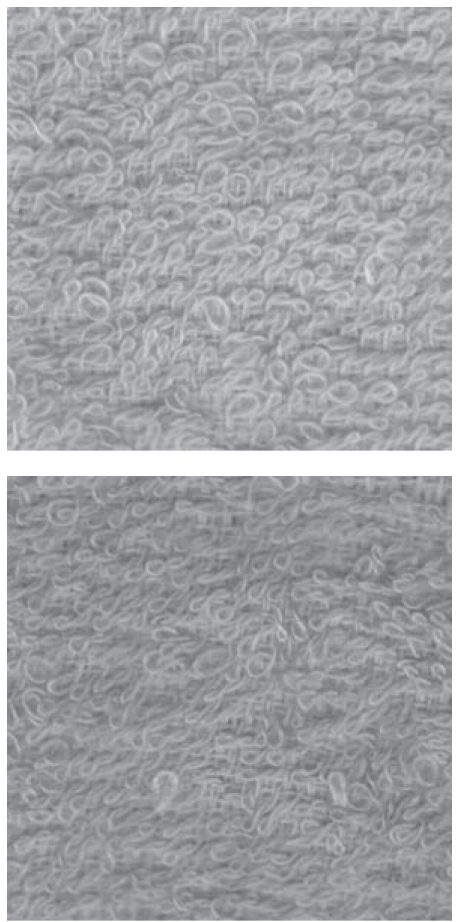

Original fabric
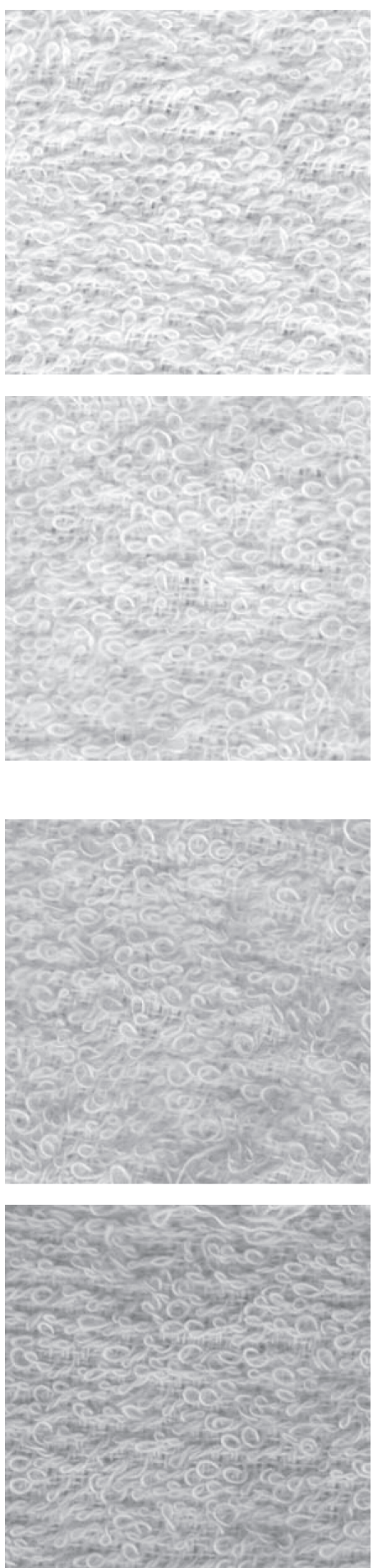

Fig. 5 The soiled and original cotton towel fabric before and after washing in LAS solution with and without hardness salts.

deposited on the soiled and original untreated fabrics, respectively.

In general, the decrease in the surface tension and the contact angle due to adsorption of surfactant are favorable to soil removal. Figure 6 shows the surface tension (data from Fig. 1) and contact angle on cellulose for the deter- 

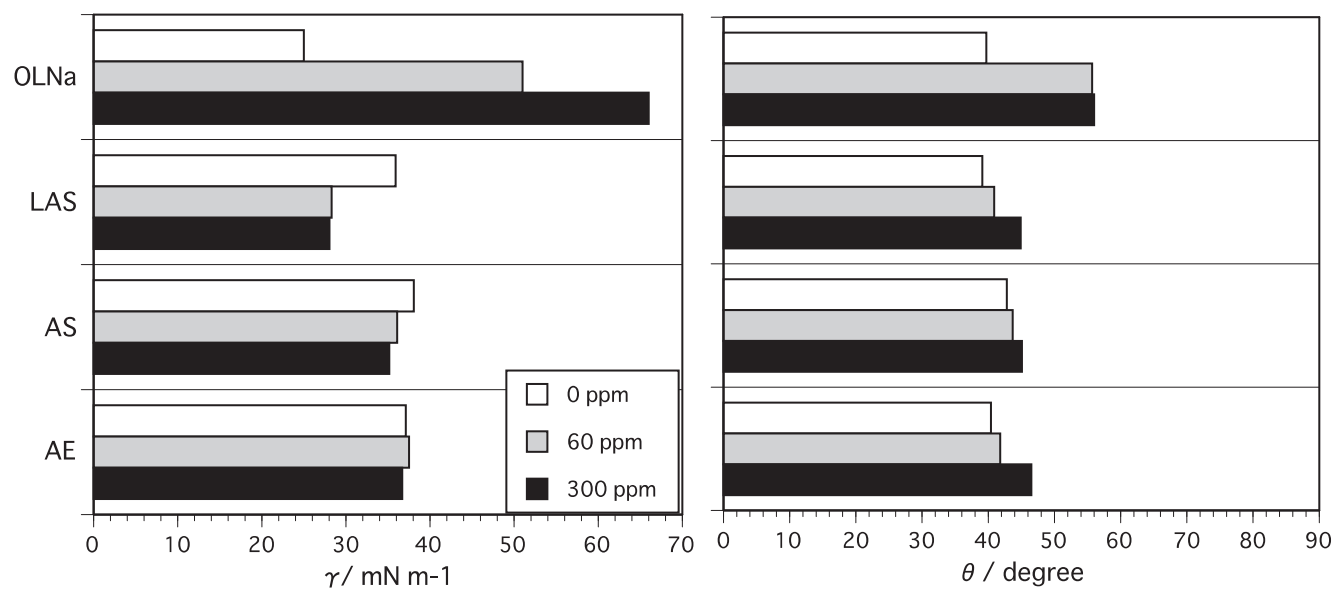

Fig. 6 Surface tension, $\gamma$, and contact angle, $\theta$, on cellulose of the surfactant solutions with and without hardness salts used for the washing test.

gent solutions used in this study. For the OLNa solution, the surface tension and contact angle increased by the addition of hardness salts, which may reduce soil removal. However, the surface tension and contact angle of the LAS, $\mathrm{AS}$, and $\mathrm{AE}$ solutions did not change much by the addition of hardness salts. Rather, the surface tension of the LAS and AS solutions decreased in the presence of hardness salts. Therefore, the effect of water hardness on the detergency power of LAS and AS was not explained in terms of surface energetics.

\subsection{Residue on cotton fiber surface after washing}

Figure 7 shows the backscattered electron images of the original and soiled cotton fabrics after washing in the LAS solutions with 0 or 300 ppm water hardness. After washing at $300 \mathrm{ppm}$, some bright spots appeared on the fiber surfaces. It seems likely that the spots can be attributed to insoluble precipitates formed by the reaction of LAS with $\mathrm{Ca}^{2+}$ or $\mathrm{Mg}^{2+11)}$.

XPS narrow spectra of $\mathrm{Mg} 1 \mathrm{~s}, \mathrm{Na} 1 \mathrm{~s}, \mathrm{Ca} 2 \mathrm{p}$, and $\mathrm{S} 2 \mathrm{p}$ of the cotton woven fabric after washing in LAS solutions are shown in Fig. 8. The Na 1s peak originating from LAS was observed after washing in the absence of hardness salts. After washing in $300 \mathrm{ppm}$ hard water significant peaks of Ca 2p and S 2p appeared, indicating that calcium and LAS were present on the fabric. A small peak of Ca $2 p$ was observed even after washing in the absence of hardness salts. This calcium content may originate from the original cotton fabric $^{11,12)}$. However, no peak of Ca $2 p$ was observed in the XPS spectra for used cotton fabrics, which had been cleaned in boiling water twice. The effect of hydrophobic impurities in cotton, such as oleic acid, should be examined in future work. From the SEM and XPS analysis, we believe that $\mathrm{Ca}(\mathrm{LAS})_{2}$ precipitates cling to cotton fiber surfaces, and remained after washing in hard water.

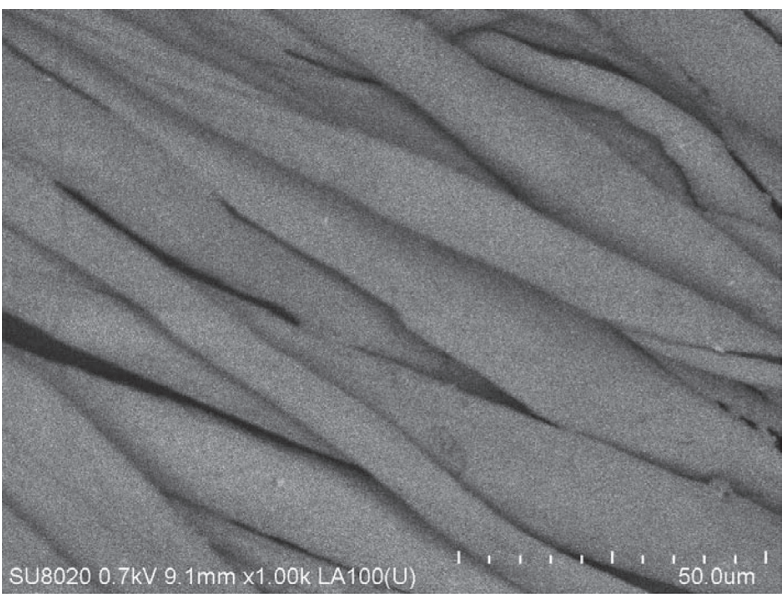

Without hardness salt (0ppm)

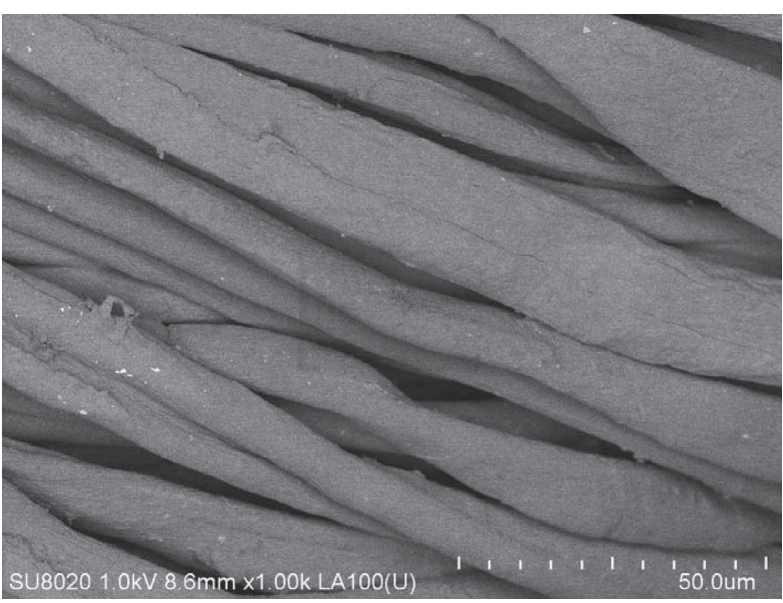

With hardness salt (300ppm)

Fig. 7 Backscattered electron images of the artificially soiled cotton woven fabric after washing in LAS solutions with and without hardness salts. 

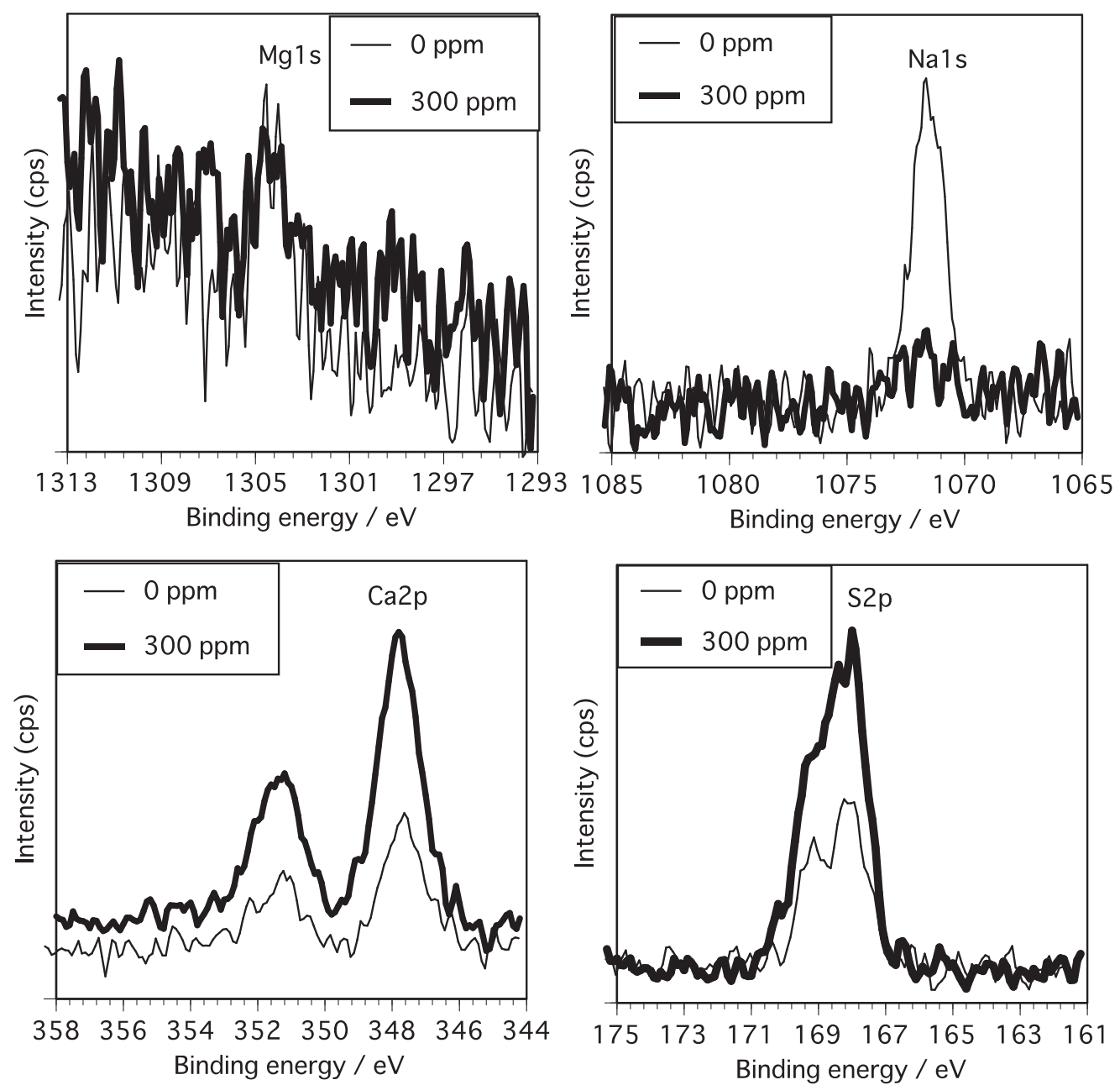

Fig. 8 XPS narrow spectra of $\mathrm{Ca} 2 \mathrm{p}, \mathrm{Mg} 1 \mathrm{~s}, \mathrm{~S} 2 \mathrm{p}$ and Na1s of cotton woven fabric after washing in LAS solutions with and without hardness salts.
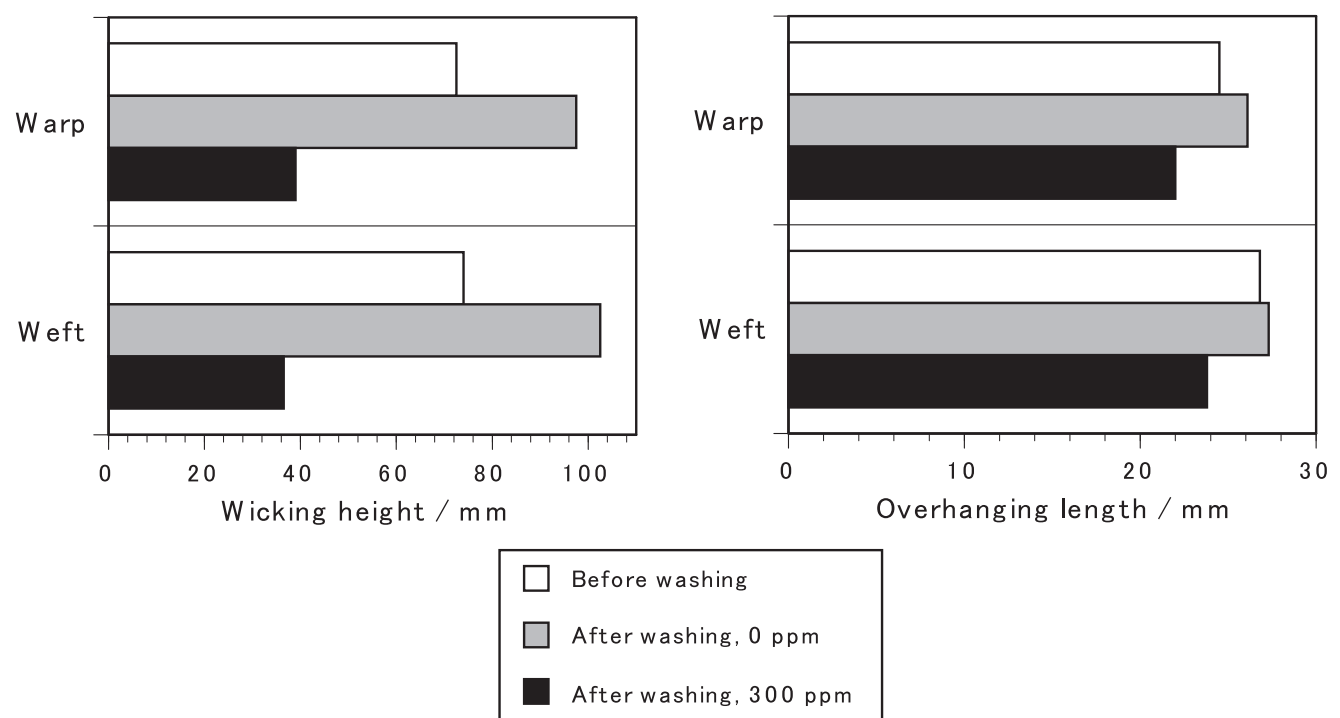

Fig. 9 Water adsorption and bending properties for cotton towel fabrics before and after washing in LAS solutions with and without hardness salts. 

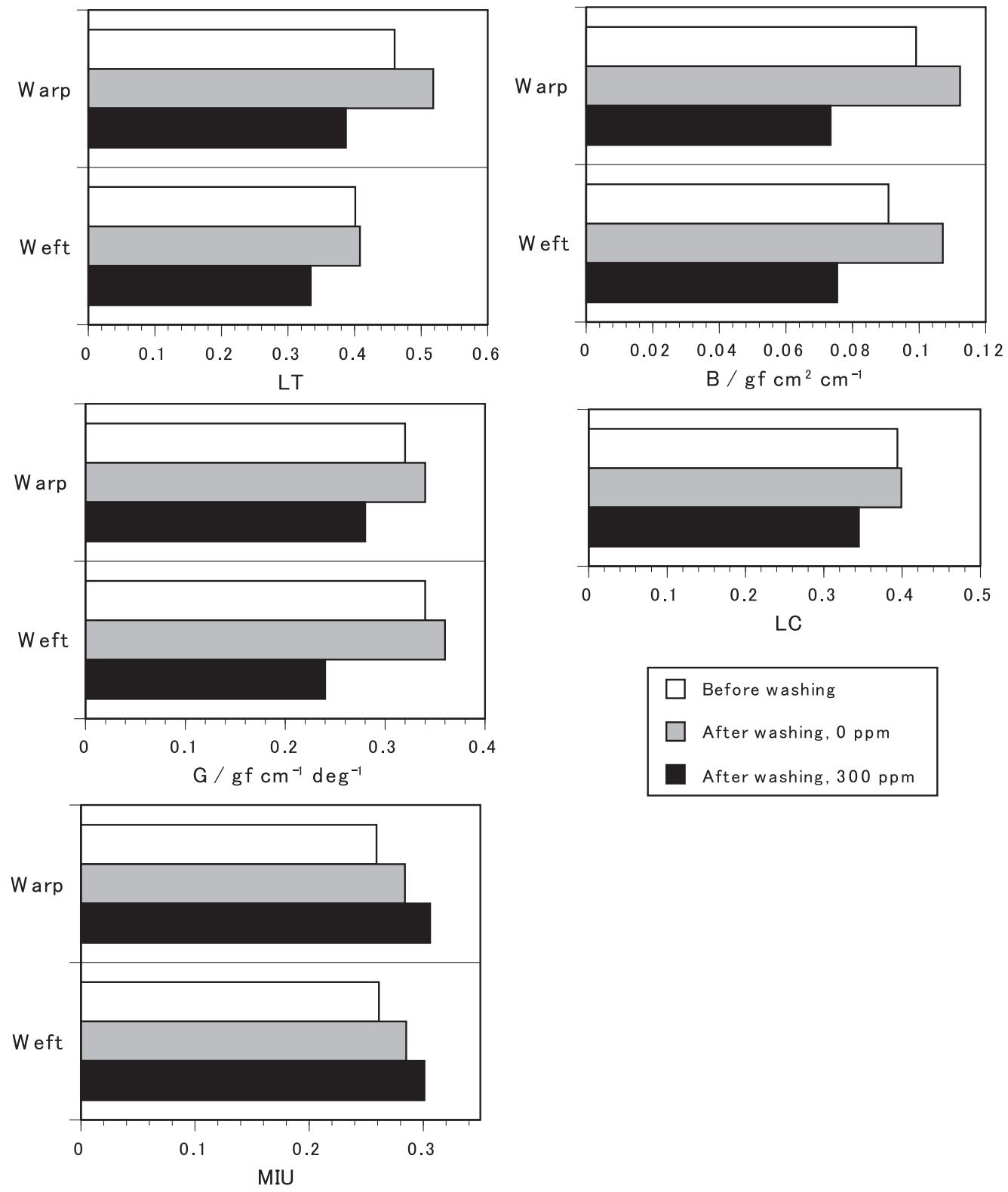

Before washing

After washing, $0 \mathrm{ppm}$

After washing, $300 \mathrm{ppm}$

Fig. 10 Linearity of load-extension curve, LT, bending rigidity, B, shear stiffness, G, linearity of compressionthickness curve, LC, and coefficient of friction, MIU determined by the FES-FB method for cotton towel fabrics before and after washing in LAS solutions with and without hardness salts.

\subsection{Cotton fabric properties after washing}

The water wicking height and the overhanging length of the cotton towel fabric before and after washing in the LAS solution are presented in Fig. 9. The wicking height significantly increased after washing in the absence of hardness salts, most likely as a result of removal of hydrophobic impurities such as waxes and pectins. However, the water wicking decreased after washing in hard water at $300 \mathrm{ppm}$. We believe that the hydrophobic $\mathrm{Ca}(\mathrm{LAS})_{2}$ precipitation deposited on the cotton fabric reduced water absorbency.

Bending resistance decreased somewhat after washing. It was expected that the deposition of the precipitate would make the fabric stiff. However, in this study the bending resistance deceased in the presence of hardness salts.

Figure 10 shows the linearity of load-extension curve, bending rigidity, shear stiffness, linearity of compressionthickness curve, and coefficient of friction, as evaluated by the KES. The LT, B, G and LC of the cotton fabric decreased after washing in the LAS solution with hardness salts. These results show that that laundering in hard water makes cotton fabric soften, which is in accordance with the bending resistance shown in Fig. 9. Although it is thought that laundering in the presence of hardness salts makes 
fabric stiff ${ }^{3,4)}$, the hydrocarbon chains of LAS remaining on fiber surfaces in the present system did not affect the fabric mechanical properties in this way. An increase in MIU was observed after washing, especially in hard water, and the precipitate deposited on the surfaces made the fabric rougher.

The adsorption of the $\mathrm{Ca}(\mathrm{LAS})_{2}$ precipitate onto the cotton fabric affects moisture wicking and mechanical properties.

\section{CONCLUSIONS}

In the presence of hardness salts, the surface tension of anionic surfactant solutions significantly changed. The surface tension increased as expected for OLNa solutions, and decreased for LAS and AS solutions. In the case of the nonionic AE solution, hardness salts did not affect the surface tension. Adding hardness salts reduced the transmittance and foamability of each anionic surfactant solution. This indicates that insoluble precipitates were formed as a result of the interaction between anionic surfactants and divalent ions. In the washing test, the soil removal decreased and redeposition increased with increasing water hardness. This tendency was in the order of LAS $>\mathrm{OLNa} \approx$ $\mathrm{AS}>\mathrm{AE}$. SEM and XPS analysis showed that $\mathrm{Ca}(\mathrm{LAS})_{2}$ precipitate clung to cotton fiber surfaces and remained after washing. By evaluation of cotton fabric properties, we found that laundering in hard water decreased water absorbency and increased fabric softness and roughness.

\section{ACKNOWLEDGEMENTS}

We wish to express our gratitude to Dr. Yasuyuki Kobayashi of Osaka Municipal Technical Research Institute, Japan, for XPS analysis and useful discussion and to Rengo Co., Ltd for providing cellulose film. Financial support from Samsung R\&D Institute Japan is gratefully acknowledged. Gratitude is expressed to the Ministry of Education, Sports, Culture, Science and Technology, Japan for a Grant-in-Aid for Scientific Research to carry out this work (grant number 26242005).

\section{References}

1) Cameron, B. A. Detergent considerations for consumers: laundering in hard water - How much extra detergent is required? J. Extension 49, 1-11 (2011).

2) Abeliotis, K.; Candan, C.; Amberg, C.; Ferri, A.; Osset, M.; Owens, J.; Stamminger, R. Impact of water hardness on consumers' perception of laundry washing result in five European countries. Int. J. Consumer Stu.
39, 60-66 (2015).

3) Okada, N.; Fujii, T.; Okuyama, H. Removal of Oily Soils (Part 2) The relation of yellowing and residue with hard ions on fabrics. J. Home Econ. Jpn. 27, 215-220 (1976).

4) Okada, N.; Fujii, T.; Minagawa, M. Removal of oily soils (Part 4) Effect of fatty acid soaps on removal of oily soils and yellowing of the fabrics washed in hard water. J. Home Econ. Jpn. 35, 322-330 (1984).

5) Matheson, K. L.; Cox, M. F.; Smith, D. L. Interaction between linear alkylbenzene sulfonate and water hardness ions. I Effect of calcium ion on surfactant solubility and implications for detergency performance. $J$. Am. Oil Chem. Soc. 62, 1391-1396(1985).

6) Cox M. F.; Matheson, K. L. Interaction between linear alkylbenzene sulfonate and water hardness ions. II . Reducing hardness sensitivety by the addition of micelle promotion agents. J. Am. Oil Chem. Soc. 62, 1396-1399 (1985).

7) Smith, D. L.; Matheson, K. L.; Cox, M. F. Interaction between linear alkylbenzene sulfonate and water hardness ions. III. Solubilization and performance characteristics of Ca(LAS) 2 . J. Am. Oil Chem. Soc. 62, 1399-1402 (1985).

8) Cohen, L.; Moreno, A.; Berna, J. L. Influence of anionic concentration and water hardness on foaming properties of a linear alkylbenzene sulfonate, J. Am. Oil Chem. Soc. 70, 75-78(1993).

9) Cohen, L; Moreno, A.; Berna, J. L. Influence of calcium concentration in the washing liquor on detergency performance of linear alkylbenzene sulfonate. J. Am. Oil Chem. Soc. 70, 79-82(1993).

10) Cohen, L.; Vergara, R.; Moreno, A.; Berna, J. L. Linear alkylbenzene sulfonate detergency behavior in water containing magnesium ions. J. Am. Oil Chem. Soc. 70, 723-726 (1993).

11) Goto-Doshida, S.; Saito M.; Nagayama, M. Effects of Calcium present in fabric and in washing liquor on detergency. J. Oleo Sci. 52, 73-82(2003).

12) Goto-Doshida, S.; Nagayama, M. Distribution of calcium ion in wash system and detergency: Calcium ion interaction with surfactants builders and cotton fabrics. J. Oleo Sci. 52, 317-329(2003).

13) Brown, D. M.; Cameron, B. A.; Meyer, S. S.; Umber, J. J. The effects of water hardness level on washing quality using commercial laundry detergents. J. Consum. Stu. Home Econ. 15, 215-222 (1991).

14) Abeliotis, K.; Candan, C.; Amberg, C.; Ferri, A.; Osset, M.; Owens, J.; Stamminger, R. Impact of water hardness on consumers' perception of laundry washing result in five European countries. Int. J. Consumer Stu. 39, 60-66 (2015).

15) Iglesias, E.; Anderez, J.; Forgiarini, A.; Salager, J.-L. A new method to estimate the stability of short-life 
forms. Colloid Surfaces A: Physicochem. Eng. Aspects 98, 167-174(1995).

16) MacLead, C. A.; Radke, C. J. A growing drop technique for measuring dynamic interfacial tension. J. Colloid Interface Sci. 160, 435-448(1993).

17) Gotoh, K.; Kobayashi, Y.; Yasukawa, A.; Ishigami, Y. Surface modification of PET films by atmospheric pressure plasma exposure with three reactive gas sources. Colloid Polym. Sci. 290, 1005-1014(2012).

18) Gotoh, K.; Harayama, K.; Handa, K. Combination effect of ultrasound and shake as a mechanical action. Ultrason. Sonochem. 22, 412-421 (2015).

19) García, M. T.; Campos, E.; Dalmau, M.; Ribosa, I.; Sánchez-Leal, J. Structure-activity relationships for asso- ciation of linear alkylbenzene sulfonates with activated sludge. Chemosphere 49, 279-286 (2002).

20) Anachkov, S. E.; Tcholakova, S.; Dimitrova, D.T.; Denkov, N.D.; Subrahmaniam, N.; Bhunia, P. Adsorption of linear alkyl benzene sulfonates on oil-water interface: Effects of $\mathrm{Na}^{+}, \mathrm{Mg}^{2+}$ and $\mathrm{Ca}^{2+}$ ions. Colloid and Surfaces A: Physicochem. Eng. Aspects 466, 18-27 (2015).

21) Fainerman, V. B.; Lylyk, S. V.; Aksenenko, E. V.; Kovalchuk, N. M.; Kovalchuk, V. I.; Petkov, J. T.; Miller, R. Effect of water hardness on surface tension and dilational visco-elasticity of sodium dodecyl sulphate solutions. J. Colloid Interface Sci. 377, 1-6(2012). 